\title{
GCU
}

Glasgow Caledonian

University

University for the Common Good

\section{Efficient depth-based scheduling MAC protoco for underwater sensor networks}

Alfouzan, Faisal Abdulaziz F; Shahrabi, Alireza; Ghoreyshi, Seyed Mohammad; Boutaleb, Tuleen

Published in:

Ninth International Conference on Ubiquitous and Future Networks (ICUFN), 2017

DOI:

10.1109/ICUFN.2017.7993915

Publication date:

2017

Document Version

Author accepted manuscript

Link to publication in ResearchOnline

Citation for published version (Harvard):

Alfouzan, FAF, Shahrabi, A, Ghoreyshi, SM \& Boutaleb, T 2017, Efficient depth-based scheduling MAC protoco for underwater sensor networks. in Ninth International Conference on Ubiquitous and Future Networks (ICUFN), 2017.., 7993915, IEEE, The 9th IEEE International Conference on Ubiquitous and Future Networks (ICUFN 2017), Milan, Italy, 4/07/17. https://doi.org/10.1109/ICUFN.2017.7993915

\section{General rights}

Copyright and moral rights for the publications made accessible in the public portal are retained by the authors and/or other copyright owners and it is a condition of accessing publications that users recognise and abide by the legal requirements associated with these rights.

Take down policy

If you believe that this document breaches copyright please view our takedown policy at https://edshare.gcu.ac.uk/id/eprint/5179 for details of how to contact us. 


\title{
Efficient Depth-Based Scheduling MAC Protocol for Underwater Sensor Networks
}

\author{
Faisal Alfouzan, Alireza Shahrabi, Seyed Mohammad Ghoreyshi, and Tuleen Boutaleb \\ School of Engineering and Built Environment \\ Glasgow Caledonian University, Glasgow, United Kingdom \\ \{Faisal.Alfouzan, A.Shahrabi, Seyed.MohammadGhoreyshi, T.Boutaleb\}@gcu.ac.uk
}

\begin{abstract}
Underwater Sensor Networks (UWSN) utilise acoustic waves with comparatively lower loss and longer range in underwater environment than electromagnetic waves. However, energy remains a challenging issue in addition to long latency, high bit error rate, and limited bandwidth. Thus, collision and retransmission should be efficiently handled at MAC layer in order to reduce the energy cost and also to improve the throughput and fairness across the network. In this paper, we therefore propose a new reservation-based distributed MAC protocol, which employs a duty cycle mechanism to address the spatial-temporal uncertainty and the hidden node problem to effectively reduce collisions and retransmissions. Our extensive simulation study reveals that our proposed protocol can efficiently handle the traffic contention to achieve significant improvement in terms of energy consumption, throughput, and fairness.

Index Terms-Underwater acoustic networks, Underwater MAC protocols, Duty cycle mechanism.
\end{abstract}

\section{INTRODUCTION}

Using acoustic signal in underwater sensor networks (UWSNs) has completely changed the design of Medium Access Control (MAC) protocols compared to that of terrestrial networks $[1,2]$. The propagation speed of acoustic signals in water is about $1500 \mathrm{~m} / \mathrm{s}$, which is five orders of magnitude lower than that of radio signals. Apart from long propagation delay, energy-efficiency is also a major concern due to difficulties of replacing or recharging batteries. The available bandwidth of acoustic channels is typically less than $15 \mathrm{kHz}$, which is much narrower compared with that of terrestrial channels. These unique acoustic communication characteristics pose challenges to underwater MAC protocol design.

The MAC protocol generally can be divided into two categories: contention-free and contention-based protocols [3]. Recently, however, most studies on MAC protocols in underwater networks head for improving the energy efficiency by using either Request-To-Send/Clear-To-Send (RTS/CTS) like handshaking approaches [4] or channel reservation techniques [5]. For the RTS/CTS method, a one-time process usually reserves the channel for only one sender-receiver pair. Although energy efficiency is extremely improved in this way, the network throughput is usually low because of the high delay in the handshaking.

In this paper, we propose a new protocol, called ED-MAC, which takes the energy efficiency, throughput, and fairness into consideration. ED-MAC schedules the transmissions and receptions of data packets at both the sender and receiver nodes to achieve the objectives of energy efficiency, high throughput, and fairness. ED-MAC consists of three phases: initial, scheduling, and normal operational phase. In the first phase, the neighbouring nodes information, such as IDs and depths, are collected by each node. In the second phase, each node reserves its own slot for its data transmission during the third phase using a depth-based timer. The spatial-temporal uncertainty and the hidden node problem are also addressed during the scheduling phase. During the normal operational phase, each node is occasionally awake to either transmit or receive data according to its schedule. The nodes can sleep when there is no packet to transmit or receive, to save more energy.

The rest of this paper is organised as follows: In Section II, we review the related work. In Section III, ED-MAC protocol is described in details. In Section IV, the performance of ED-MAC protocol is evaluated and compared against those of UWAN-MAC and T-Lohi protocols through simulations. Finally, Section $\mathrm{V}$ concludes the paper.

\section{RELATED WORK}

Underwater MAC is a core component of the underwater acoustic network system to enable multiple nodes to share the broadcast channel efficiently. Underwater MAC protocols can generally be classified into two categories: contention-free and contention-based. TDMA-based protocols, such as ST-MAC [6] and STUMP [7], are typical contention-free MAC protocol for UWSNs. However, they require centralized scheduling, which results in either excessive control overhead or sluggish responses to network dynamics.

In contrast, the contention-based MAC protocols can be more flexible and responsive to dynamic network topologies and therefore are more suitable for UWSNs. This type of protocols can be further classified into two classes, namely handshaking based and random based MAC protocols [8, 9]. Protocols in different classes have distinctive performance on throughput, delay or energy efficiency. In the first class, handshaking, sending and receiving nodes exchange some control packets before sending data packets trying to avoid any possible collision. In the second class, random based, nodes attempt randomly to access the medium. When a data packet arrives at a receiver, if there is no packet coming from other nodes, the receiver can receive this packet successfully. However, most of the efforts of the MAC protocol design for UWSNs have focused on the handshaking class such as 
DACAP [10] and S-FAMA [4]. Some variances based on the handshaking class have also been proposed [11-13].

In [10], a Distance-Aware Collision Avoidance Protocol (DACAP) is a handshaking based protocol, which combines carrier sensing (CS) and an exchange of RTS/CTS control packets before transmitting data, but it does not require any synchronisation between nodes. However, exchanging RTS/CTS control packets between the source and destination to eliminate data packet collisions, consumes a large amount of energy in underwater sensor networks. Slotted Floor Acquisition Multiple Access (S-FAMA) [4] is another handshaking based protocol, which combines both carrier sensing (CS) and a dialogue between the source and receiver before data transmission. During the first dialogue, control packets are exchanged between the source node and the destination node to avoid multiple transmissions at the same time. Although SFAMA avoids DATA packet collisions without relying on the packet size, it requires clock synchronisation between nodes which is difficult to achieve in UWSNs.

According to the random based class, a sender transmits packets randomly or after a simplified one-way contention. Aloha with advanced notification (Aloha-AN) is a random based protocol, which consists of sending a short data packet prior to the actual data transmission with information on the sender and the intended receiver [14]. A similar approach called T-Lohi is proposed in [15], which uses a tone-based contention mechanism to detect collisions. To make T-Lohi work, the tone signal is assumed short enough to eliminate collisions.

UWAN-MAC [16] is another random based protocol, which leverages local synchronisation to arrange the time-line of each node for energy efficiency improvement. It provides an appropriate method by increasing the sleep mode rather than the idle listening mode. This is based on the fact that sleep mode consumes less energy than the idle listening mode. In particular, UWAN-MAC is an energy efficient MAC protocol designed for underwater network. For instance, when the sender transmits a packet, it actually informs the receiver when it is supposed to send the next packet, and then the neighbours will overhear the packet to avoid the possible collisions [17]. The main disadvantage of this protocol is that the spatialtemporal uncertainty and the hidden terminal problem are not addressed; therefore, it consumes more energy because of the collisions and retransmissions.

\section{EFFicient Depth-BASEd MAC PROTOCOL}

In this section, we first brief the basic ideas of ED-MAC. We then describe each phase in details.

\section{A. Overview}

Efficient Depth-Based (ED-MAC) is a reservation-based MAC protocol. It employs a duty cycle mechanism by assigning time slots to every individual node in the network in a distributed manner. The primary goal is to reduce the energy consumption by using a wakeup scheduling scheme; nodes are awake in some slots to transmit or receive data and are asleep
TABLE I: Notations

\begin{tabular}{c|c}
\hline \hline Terms & Definition \\
\hline$N$ & underwater sensor node \\
$B_{p}$ & Beacon packet \\
$S_{p}$ & Schedule packet \\
$N . I D$ & Node's ID \\
$N . d e p t h$ & Node's depth measured by water pressure \\
$N_{t}$ & Neighbouring table \\
$D P L$ & Depth Priority List \\
$T_{\text {wake-up }}$ & The wake-up times of a node \\
$M_{\text {Depth }}$ & The depth of the network \\
$N_{\text {Depth }}$ & A node depth in the network \\
$T_{b}$ & Predefined fixed value for the initial phase \\
$T_{\text {Delay }}$ & Predefined maximum delay \\
$T_{s c h}$ & Scheduling timer of each node \\
$R_{t}$ & Length of each round \\
$D_{r}$ & Data rate \\
$N_{s}$ & Number of slots \\
$N_{s s}$ & Number of sub-slots \\
$N_{\text {max }}$ & Maximum number of nodes per neighbourhood \\
$L_{s}$ & Length of each slot \\
$L_{s s}$ & Length of each sub-slot \\
$\triangle_{\text {DataProp }}$ & Propagation delay \\
$R_{\text {time }}$ & Receiving time of data packet \\
$G_{t}$ & Guard time \\
$T_{r}$ & Transmission range \\
$U_{s}$ & Speed of sound in water \\
\hline \hline
\end{tabular}

over the remaining slots. The possibility of collision is very slim and limited to a very specific scenario. To remove even this slight chance, every slot is divided into a number of subslots. These sub-slots are selected randomly to avoid collision across the network. The length of each sub-slot is equal to a signal propagation delay plus a small guard time. ED-MAC trades off latency for energy efficiency and fairness and hence provide the flexibility to be utilised for various energy-critical applications.

Nodes in the network operate in three phases; namely initial, scheduling, and normal operational phase. All network nodes operate asynchronously during each phase but share a common clock to start and end each phase together. To eliminate the effect of any clock drift that may occur over a long period of time, a guard time is applied where appropriate.

\section{B. Initial Phase}

At the deployment time, the start time of the initial phase for each node has been set. During this phase, every node randomly broadcasts its beacon packet to its neighbourhood. The beacon packet includes the ID and depth of the sender. The purpose of this phase is to exchange the ID and depth between neighbouring nodes, as shown in Algorithm 1, which can be used at each node to generate neighbouring table,

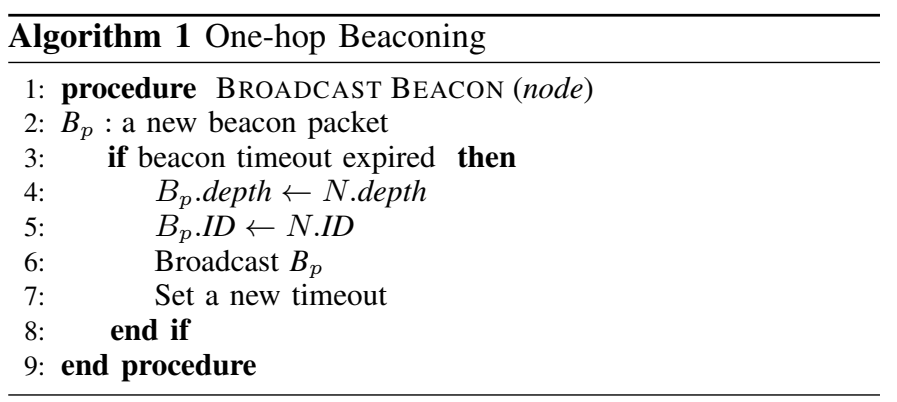


$N_{t}$. Upon receiving a beacon packet, every node immediately updates its $N_{t}$. The length of this phase, $T_{b}$, is set to a predefined fixed value for all nodes. It is a function of the transmission range of each node, $T_{r}$, and the maximum number of nodes per neighbourhood, $N_{\max }$. It should be long enough to let them to create their own one-hop neighbouring tables with accurate information. However, the length of this phase is very short compared to that of the third phase.

\section{Schedule Phase}

Using the information stored in the neighbouring table, $N_{t}$, during the first phase, every node in the network needs to reserve a unique slot for itself to use during the third phase for data transmission. It needs to know which slots are reserved by its one-hop neighbouring nodes to adjust its wakeup time as well. Hence, the goal of the second phase is to schedule the wakeup and sleep times at each node. A depth based timer is used to prioritise nodes when reserving a slot. This allows a node located in deeper area to reserve a slot sooner than its above neighbouring nodes. The value of this timer at each node is given by

$$
T_{\text {sch }}=\left(\frac{M_{\text {Depth }}}{M_{\text {Depth }}+N_{\text {Depth }}} * T_{\text {Delay }}\right)-\frac{T_{\text {Delay }}}{2}
$$

where $M_{\text {Depth }}$ is the depth of the network area and $N_{\text {Depth }}$ is a node depth in the network. $T_{\text {Delay }}$ is a predefined fixed value to provide more granularity. The value of $T_{\text {Delay }}$ depends also on the density of the nodes in underwater area. It should be long enough to avoid collisions between neighbouring nodes, which are vertically very close to each other, during the scheduling phase. For instance, the deeper network should set $T_{\text {Delay }}$ with a higher value in order to prevent any conflict between neighbouring nodes.

A node before broadcasting its schedule packet, $S_{p}$, extracts the neighbouring nodes with lower depth than itself from $N_{t}$ and place them in depth priority list, $D P L$, in order of their depths. Thereafter, each node broadcasts its $S_{p}$ to its neighbouring nodes using depth-based timer. The $S_{p}$ includes the reserved sender slot and its $D P L$. Upon receiving a $S_{p}$, every node compares its $N_{t}$ with the received $D P L$ to detect the hidden nodes.

This procedure is continuously executed by nodes in order of their depths and all nodes gradually reserve their own transmission slots while also informing their neighbouring nodes about that. The length of the scheduling phase is a constant value, $\left(T_{\text {Delay }} / 2\right)$, set at the deployment time based on the application requirement. It is set to a small value for sparse and shallow scenarios and set to large values for deep and dense networks. Either way, the length of this phase is smaller than that of the third phase by a few order of magnitude. Algorithm 2 shows how a node reserves a slot and provide other neighbouring nodes with some information including its reserved slot and a list of some of its neighbouring nodes.

\section{Normal Operational Phase}

In this phase, nodes wake up and sleep periodically. In other words, nodes are awake in some slots and asleep during

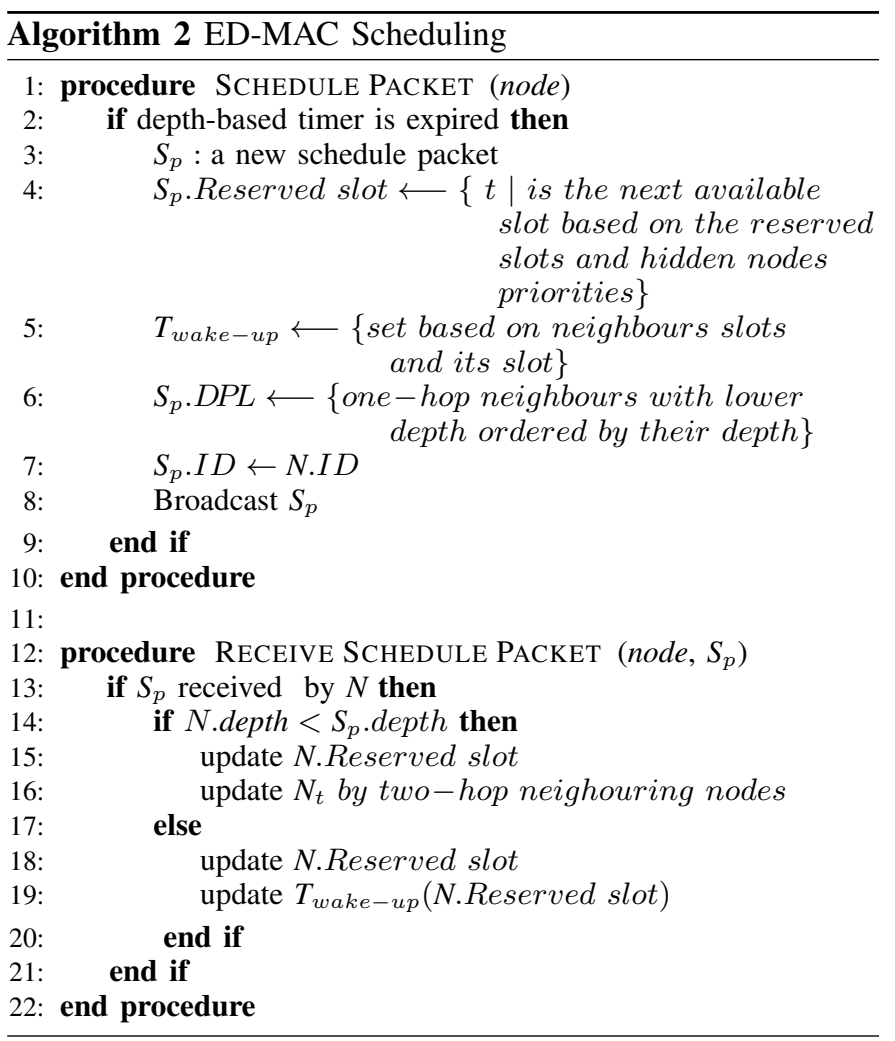

the remaining slots when there is no data transmission or reception. This phase is divided into some rounds and each round is consisted of a number of slots. Every slot has also a number of sub-slots. These slots are reserved by the nodes in the scheduling phase. The sub-slots are selected randomly to avoid the possibility of collisions which can be caused by spatial-temporal uncertainty and hidden terminal problem. The length of each round, $R_{t}$, has a reverse relationship with data rate, $D_{r}$, which is presented in terms of packet per second. The higher the data rate the shorter round time and, hence, the shorter sleeping time. The duration of each round time is given by

$$
R_{t}=\frac{1}{D_{r}}
$$

We divide $R_{t}$ into a number of slots, $N_{s}$. The number of slots per round is proportional to the maximum number of nodes in a neighbourhood. To exclude the possibility of concurrent data transmission from nodes located outside of a one-hop neighbourhood and the node within the neighbourhood, the number of slots are doubled per round. We derive $N_{s}$ by using:

$$
N_{s}=2 \times N_{\max }
$$

where $N_{\max }$ is the estimated maximum number of nodes in a particular one-hop neighbourhood. The length of each slot, $L_{s}$, can be calculated using:

$$
L_{s}=\frac{R_{t}}{N_{s}}
$$

Our proposed algorithm is almost a collision-free algorithm for most of scenarios. However, one slot might be reserved, under a very specific circumstances, by two hidden nodes 


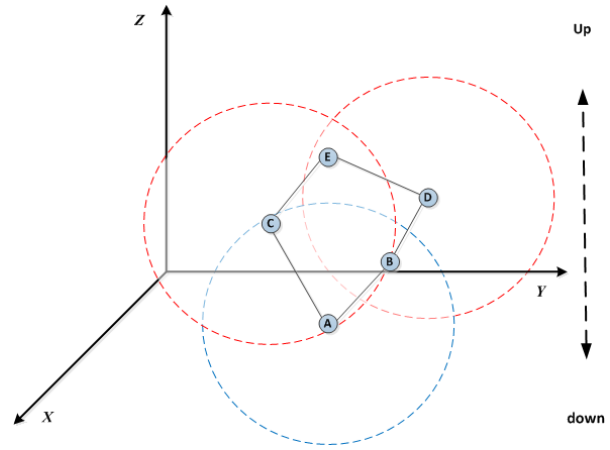

Fig. 1: A very specific scenario leading to a potential collision. This has been addressed in ED-MAC.

which both are neighbouring nodes of another node with lower depth. This specific scenario is illustrated in Fig. 1. Node $A$ has reserved slot $n, 1 \leq n \leq N_{s}$ and, consequently, slots $(n+1) \bmod N_{s}$ and $(n+2) \bmod N_{s}$ are reserved by its neighbouring nodes $B$ and $C$, respectively. As node $D$ is not aware of the slot number reserved by node $C$, it reserves the same slot, i.e. slot $(n+2) \bmod N_{s}$. Now, transmitting a packet by nodes $C$ and $D$ result in a collision in node $E$.

To address this issue, the concept of sub-slot is introduced here. Each slot is divided into a number of equal size sub-slots. To transmit a packet, a node randomly selects one sub-slot out of $N_{s s}$ available sub-slots to transmit its packet. The length of every sub-slot is longer than propagation delay to ensure that a packet is entirely received at the destination before starting of data transmission by another node. The length of each sub-slot is given by

$$
L_{s s}=\triangle_{\text {DataProp }}+R_{\text {time }}+G_{t}
$$

where $R_{\text {time }}$ indicates the receiving time of data packet. $G_{t}$ denotes the guard time, which is used to ensure that distinct transmissions do not interfere with one another. Also, $\triangle_{\text {DataProp }}$ denotes the propagation delay of a transmitted packet. The propagation delay, $\triangle_{\text {DataProp }}$, can also be calculated using:

$$
\triangle_{\text {DataProp }}=\frac{T_{r}}{U_{s}}
$$

where $T_{r}$ denotes the transmission range, and $U_{s}$ indicates the speed of sound in water. Finally, the number of sub-slots, $N_{s s}$, can also be calculated using the following equation:

$$
N_{s s}=\frac{L_{s}}{L_{s s}}
$$

\section{PERformance Evaluation}

In this section, we first discuss the implementation of our protocol, ED-MAC, in the Aqua-Sim underwater simulation. We also evaluate the performance of ED-MAC protocol and compare it with UWAN-MAC and T-Lohi protocols. We then present and analyse the simulation results.

\section{A. Implementation}

We implement ED-MAC in Aqua-Sim, an NS-2 based simulator for underwater sensor networks. Unless specified otherwise, we use the following parameters in the simulations. The power consumption on transmission mode is 2 Watts; the power consumption on receive mode is 0.75 Watts; and the power consumption on sleep mode is $8 \mathrm{~mW}$. The data packet size is set to 2000 bits and the control packet size is set to 100 bits. The bit rate is $10 \mathrm{Kbps}$ and the maximum transmission range is 100 meters. The bandwidth is $100 \mathrm{Kbps}$. This simulation shows how ED-MAC protocol performs in underwater acoustic networks. In our simulation, we consider two parameters: data generated rate and number of nodes. In the first parameter, all nodes are distributed in a $10000 \mathrm{~m}^{2} \times$ $200 \mathrm{~m}$ area. We deploy 10 nodes into the network and compare the network successful delivery ratio, energy consumption, and fairness index of ED-MAC, UWAN-MAC, and T-Lohi protocols with different data generated rate in this area. In the second parameter, however; all nodes are distributed within an area of $62500 \mathrm{~m}^{2} \times 500 \mathrm{~m}$, while increasing the number of nodes until 100 nodes in this narrow area. We consider $T_{b}$ as 30 seconds and $T_{\text {Delay }}$ as 60 seconds in our simulation setup. In this set of simulations, we investigate the performance of ED-MAC protocol and compare it with UWAN-MAC and TLohi protocols.

\section{B. Performance Metrics}

We define three metrics to compare the performance of EDMAC, UWAN-MAC, and T-Lohi: Successful delivery ratio, Energy consumption, and the Fairness index.

1) Successful delivery ratio: is defined as the ratio of the number of packets received successfully to the total number of packets generated in the network.

2) Energy consumption: is obtained by dividing the overall energy consumption in the network by the successfully delivered data packets, which is measured in joules per packet.

3) Fairness index: is a key performance of MAC protocol, which affects the normal operation and survival time of UWSNs. To evaluate the fairness of among ED-MAC, UWANMAC, and T-Lohi protocols, we adopt the Jain's Fairness Index define in [18]:

$$
\text { Fairness Index }=\frac{\left(\sum x_{i}\right)^{2}}{\left(n \cdot \sum x_{i}^{2}\right)}
$$

where $x_{i}$ denotes the throughput of node $i$ and $n$ denotes the number of nodes in the network. The ranges index value between 0 and 1 of a given metric could be used suitably as a measure of fairness, when the index value is closer to 1 , this indicates that the protocol has a good fairness and vice versa.

\section{Simulation results}

The performance of ED-MAC is compared with UWANMAC and T-Lohi through simulations. For each test, the results are averaged over 50 runs, with a randomly generated topology in each run. The total simulation time for each run is 3600 seconds. In the simulations, two parameters are deployed in order to perform ED-MAC, UWAN-MAC, and T-Lohi protocols in underwater networks. Firstly, 10 nodes are uniformly deployed into the network and successful delivery ratio, energy 


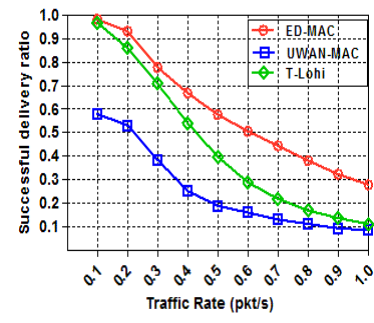

Fig. 2: Successful delivery ratio vs traffic rate

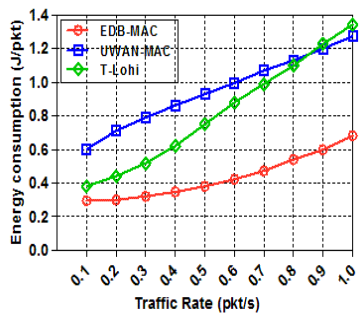

Fig. 3: Energy consumption vs traffic rate

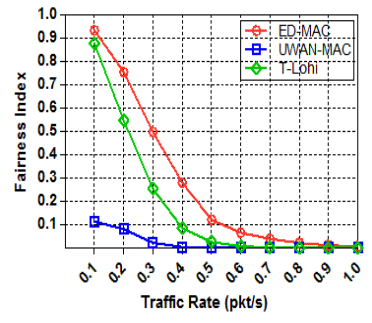

Fig. 4: Fairness index vs traffic rate consumption, and fairness index of ED-MAC, UWAN-MAC, and T-Lohi protocols are performed by changing the datagenerated rate. Secondly, the traffic rate $\lambda$ is fixed to 0.25 by changing the node density. The nodes are randomly deployed in a large narrow area and the transmission range fixed to 100 meters. This simulation shows how these three MAC protocols perform in practical underwater networks.

In the first set of simulations, we compare the successful delivery ratio, energy consumption, and fairness index with the traffic rate in all three MAC protocols. The number of nodes is kept fixed to 10; and the traffic rate increases from 0.1 to 1 . Fig. 2 examines the successful delivery ratio of three protocols as a function of traffic rate. The successful delivery ratio of ED-MAC outperforms that of other two MAC protocols, T-Lohi and UWAN-MAC. This is because these two protocols cannot detect the hidden terminal problems which lead to more packet loss and collisions. At a low traffic rate, the successful delivery ratio of ED-MAC and T-Lohi almost achieves $100 \%$, while UWAN-MAC only reaches $60 \%$. This is due to the inefficient scheduling of UWAN-MAC, which causes considerably more collisions and retransmissions. This is also because ED-MAC and T-Lohi exploit space-time uncertainty and high latency to detect collisions. When the traffic is further increased, however, T-Lohi shows a fast degradation of the successful delivery ratio due to an increasing number of collisions of tone packets. The performance of ED-MAC in this simulation is far better than that of T-Lohi and UWANMAC, as ED-MAC employs priority approach based on the depth of each sensor node to schedule the transmission and also to avoid data collisions. Fig. 3 demonstrates that ED-MAC is much more energy efficient in this simulation than T-Lohi and UWAN-MAC as a function of traffic rate. This is mainly because, as previously mentioned, T-Lohi and UWAN-MAC cannot detect the hidden terminal problems which lead to more collisions and retransmissions. Collisions and retransmissions should thus be reduced in order to reduce energy consumption and improve throughput. At a low traffic rate, ED-MAC is still more energy efficient than other protocols due to its lower receiving overhead and less idle overhead per data packet. Another reason for ED-MACs superior efficiency is that it has a compatible schedule based on its depth criteria, which helps to avoid the possibility of collisions. As the traffic rate increases, T-Lohi shows a rapid rise of the energy consumption per packet due to an increasing number of collisions of control packets. It is more interesting to observe that when the traffic rate increases, the energy consumption of ED-MAC also slightly increases, although it still has a higher network throughput than T-Lohi and UWAN-MAC protocols. Fig. 4 shows the result of an experimental setup consisting of ten nodes that are run for an hour to strenuously test the fairness of the three protocols. First, it is observed that the ED-MAC protocol exhibits a high fairness index of almost $93 \%$, which then decreases across all the traffic rates. In comparison, the fairness index of T-Lohi and UWAN-MAC (within 0.1 data rate) achieves nearly $88 \%$ and $12 \%$ respectively. When the traffic rate further increases, the fairness index of all protocols significantly decreases. ED-MAC protocol achieves higher fairness of transmission and throughput than other protocols due to the channel reservation, which is based on the depth criteria. Another reason for the considerably lower fairness of T-Lohi and UWAN-MAC compared to ED-MAC is temporal and spatial reuse.

The second set of simulations studies how the node density affects the performance of the three different MAC protocols. In this experiment, the traffic rate $\lambda$ is set to 0.25 and the number of nodes increases from 10 to 100 . As can be seen from Fig. 5, the successful delivery ratio of all three protocols is inversely proportional to the number of nodes. With 10 nodes, ED-MAC reaches $88 \%$ compared to $78 \%$ and $45 \%$ for T-Lohi and UWAN-MAC respectively. This is due to the specific benefits of ED-MAC, such as high scalable scheduling, consequently it can handle more packets than either T-Lohi or UWAN-MAC. When the number of nodes is increased, T-Lohis successful delivery ratio rapidly reduces due to an increasing number of collisions of tone packets. The impact of increasing the number of nodes on the successful delivery ratio in T-Lohi is more profound. Still more interesting, however, is that UWAN-MAC only achieved $45 \%$ of the successful delivery ratio (within 10 nodes) which is much lower than the successful delivery ratio of either ED-MAC or T-Lohi. This is because UWAN-MAC is designed to only use one control packet across the network. As shown in Fig. 6, the energy consumption of the three protocols is inversely proportional to the number of nodes. As the number of nodes increases, the energy consumption increases correspondingly. This is because when the number 


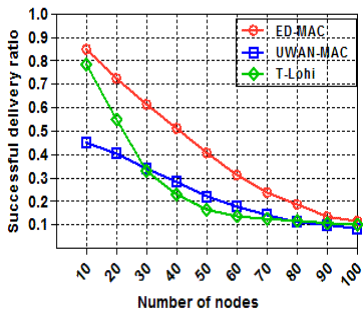

Fig. 5: Successful delivery ratio vs node density

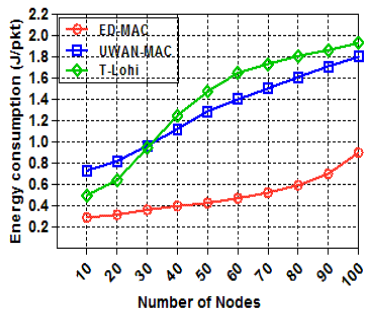

Fig. 6: Energy consumption vs node density

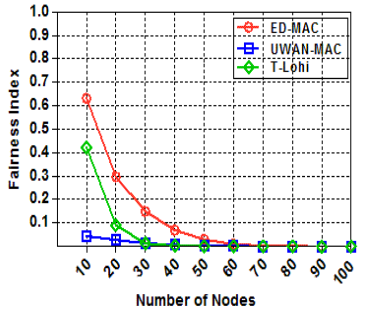

Fig. 7: Fairness index vs node density of nodes increases, more nodes are involved, therefore there is more intensive competition to access the channel. ED-MAC consumes the lowest energy among the three protocols because it adopts energy conservation measures by considering hidden terminal and spatial-temporal uncertainty problems. These considerations reduce the number of collisions, thus ED-MAC reduces energy consumption and improves throughput. As the node density increases, T-Lohi consumes significantly more energy per packet due to an increasing number of collisions. Specifically, ED-MAC consumes on average only 53.6\% of the energy consumption of T-Lohi and $50.1 \%$ of the energy consumption of UWAN-MAC. Fig. 7 shows the fairness index of all three protocols. As the number of nodes increases, the fairness index of all protocols declines considerably. This is because the network congestion reduces the fairness in all three protocols. Due to the large delays in the underwater acoustic network, the distance between nodes becomes a key factor in the competitive channel. In contrast, the fairness index of ED-MAC (within 10 nodes) is higher than both T-Lohi and UWAN-MAC by approximately $21 \%$ and $59 \%$ respectively. This is because that the latter protocols cannot avoid the hidden terminal problem, which lead to increase the number of collisions and retransmissions. Moreover, UWANMAC involves unknown propagation delays, which affects its fairness compared to other two protocols.

\section{Conclusion}

In this paper, we have proposed an efficient reservationbased distributed MAC protocol (ED-MAC) for underwater sensor networks. ED-MAC uses a duty cycle mechanism which can reduce energy consumption as well as improving throughput and fairness by handling the traffic contention effectively. The spatial-temporal uncertainty as well as the hidden node problem have also been addressed. Using an extensive simulation study, the performance of ED-MAC has been compared against those of two other protocols from the same category recently reported in the literature. The results have demonstrated the improvement achieved in terms of the successful delivery ratio, energy consumption, and fairness index with varying traffic rates and number of nodes.

\section{REFERENCES}

[1] C.-C. Hsu, M.-S. Kuo, C.-F. Chou, and K. C.-J. Lin, "The elimination of spatial-temporal uncertainty in underwater sensor networks," IEEE/ACM Transactions on Networking, vol. 21, no. 4, pp. 1229-1242, 2013.
[2] L. Hong, F. Hong, Z. Guo, and Z. Li, "Ecs: Efficient communication scheduling for underwater sensor networks," Sensors, vol. 11, no. 3, pp. 2920-2938, 2011.

[3] M. Xu, G. Liu, and J. Guan, "Towards a secure medium access control protocol for cluster-based underwater wireless sensor networks," International Journal of Distributed Sensor Networks, 2015.

[4] M. Molins and M. Stojanovic, "Slotted fama: a mac protocol for underwater acoustic networks," in OCEANS 2006-Asia Pacific, pp. 1-7, IEEE, 2007.

[5] P. Xie and J.-H. Cui, "R-mac: An energy-efficient mac protocol for underwater sensor networks," in Wireless Algorithms, Systems and Applications, 2007. WASA 2007. International Conference on, pp. 187198, IEEE, 2007.

[6] C.-C. Hsu, K.-F. Lai, C.-F. Chou, and K.-J. Lin, "St-mac: Spatialtemporal mac scheduling for underwater sensor networks," in INFOCOM 2009, IEEE, pp. 1827-1835, IEEE, 2009.

[7] K. Kredo II, P. Djukic, and P. Mohapatra, "Stump: Exploiting position diversity in the staggered tdma underwater mac protocol," in INFOCOM 2009, IEEE, pp. 2961-2965, IEEE, 2009.

[8] K. Chen, M. Ma, E. Cheng, F. Yuan, and W. Su, "A survey on mac protocols for underwater wireless sensor networks," IEEE Communications Surveys \& Tutorials, vol. 16, no. 3, pp. 1433-1447, 2014.

[9] S. Climent, A. Sanchez, J. V. Capella, N. Meratnia, and J. J. Serrano, "Underwater acoustic wireless sensor networks: advances and future trends in physical, mac and routing layers," Sensors, vol. 14, no. 1, pp. 795-833, 2014.

[10] B. Peleato and M. Stojanovic, "Distance aware collision avoidance protocol for ad-hoc underwater acoustic sensor networks," IEEE Communications Letters, vol. 11, no. 12, 2007.

[11] C. Petrioli, R. Petroccia, and M. Stojanovic, "A comparative performance evaluation of mac protocols for underwater sensor networks," in OCEANS 2008, pp. 1-10, IEEE, 2008.

[12] N. Chirdchoo, W.-s. Soh, and K. C. Chua, "Ript: A receiver-initiated reservation-based protocol for underwater acoustic networks," IEEE Journal on Selected Areas in Communications, vol. 26, no. 9, 2008.

[13] Y. Noh, P. Wang, U. Lee, D. Torres, and M. Gerla, "Dots: A propagation delay-aware opportunistic mac protocol for underwater sensor networks," in Network Protocols (ICNP), 2010 18th IEEE International Conference on, pp. 183-192, IEEE, 2010.

[14] N. Chirdchoo, W.-S. Soh, and K. C. Chua, "Aloha-based mac protocols with collision avoidance for underwater acoustic networks," in INFOCOM 2007. 26th IEEE International Conference on Computer Communications. IEEE, pp. 2271-2275, IEEE, 2007.

[15] A. A. Syed, W. Ye, and J. Heidemann, "T-lohi: A new class of mac protocols for underwater acoustic sensor networks," in INFOCOM. The 27th Conf. on Computer Communications, pp. 231-235, IEEE, 2008.

[16] M. K. Park and V. Rodoplu, "Uwan-mac: An energy-efficient mac protocol for underwater acoustic wireless sensor networks," IEEE journal of oceanic engineering, vol. 32, no. 3, pp. 710-720, 2007.

[17] F. Alfouzan, A. Shahrabi, S. M. Ghoreyshi, and T. Boutaleb, "Performance comparison of sender-based and receiver-based scheduling mac protocols for underwater sensor networks," in Network-Based Information Systems (NBiS), 2016 19th International Conference on, pp. 99-106, IEEE, 2016.

[18] R. Jain, D.-M. Chiu, and W. R. Hawe, A quantitative measure of fairness and discrimination for resource allocation in shared computer system, vol. 38. ERL, Digital Equipment Corporation Hudson, MA, 1984. 